\title{
Effect of flowable thrombin-containing collagen-based hemostatic matrix for preventing pancreatic fistula after pancreatectomy: A randomized clinical trial
}

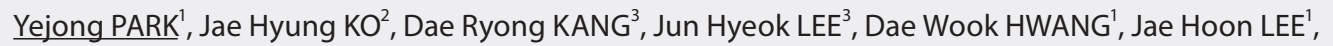
Woohyung LEE', Jaewoo KWON', Si-Nae PARK², Ki-Byung SONG', Song Cheol KIM¹

'Division of Hepato-Biliary Pancreatic Surgery, Department of Surgery, University of Ulsan College of Medicine \& Asan Medical Center, Seoul, Korea

${ }^{2}$ Regenerative Medicine Research Center, Seoul, Korea

${ }^{3}$ Department of Precision Medicine, Wonju College of Medicine, Yonsei University, Wonju, Korea

Introduction: The aim of this study was to evaluate the safety and efficacy of a flowable hemostatic matrix, and their effect for postoperative pancreatic fistula (POPF) after pancreatectomy.

Methods: This was a randomized, clinical, single-center, single-blind (participant), non-inferiority, phase IV, and parallel-group trial. The primary endpoint was the incidence of POPF. The secondary endpoints were risk factors for POPF, drain removal days, incidence of complication, 90-day mortality, and length of hospital stay.

Results: This study evaluated a total of 54 patients, with 26 patients in the intervention group (flowable hemostatic matrix) and 27 patients in the control group (thrombin-coated collagen patch). POPF was more common in the control group than in the intervention group (59.3\% vs. $30.8 \%, p=0.037)$. Among participants who underwent distal pancreatectomy (DP), POPF (33.3\% vs. $92.3 \%, p=0.004)$ and clinically relevant POPF ( $8.3 \%$ vs. $46.2 \%, p=0.027)$ were more common in the control group. A multivariate logistic regression model identified flowable hemostatic matrix use $(p=0.029)$ as an independent negative risk factor for POPF.

Conclusions: Flowable hemostatic matrix application is a simple, feasible, and effective method of preventing POPF after pancreatectomy, especially for patients with DP. 